\title{
Scalp involvement in pemphigus: a prognostic marker
}

\author{
Marta Sar-Pomian¹, Marek Konop ${ }^{1}$, Kamila Gala², Lidia Rudnicka ${ }^{1}$, Malgorzata Olszewska ${ }^{1}$ \\ ${ }^{1}$ Department of Dermatology, Medical University of Warsaw, Warsaw, Poland \\ ${ }^{2}$ Department of Immunology, Transplantology and Internal Diseases, Medical University of Warsaw, Warsaw, Poland \\ Adv Dermatol Allergol 2018; XXXV (3): 293-298 \\ DOI: https://doi.org/10.5114/pdia.2017.71267
}

\begin{abstract}
Introduction: Scalp involvement in the course of pemphigus is observed in $16-60 \%$ of patients.

Aim: To determine the prognostic significance of scalp involvement in pemphigus vulgaris and pemphigus foliaceus. Material and methods: A total of 75 patients (46 with pemphigus vulgaris, 29 with pemphigus foliaceus) were included into this prospective study. The following clinical data were analyzed: Pemphigus Disease Area Index, time to complete clinical remission and duration of complete clinical remission. Indirect immunofluorescence and enzyme-linked immunosorbent assay were performed to monitor serum pemphigus antibodies.

Results: Scalp involvement was observed in 30/46 (65.2\%) patients with pemphigus vulgaris and 28/29 (96.6\%) patients with pemphigus foliaceus. A positive correlation was found between scalp involvement and general disease severity as measured by the Pemphigus Disease Area Index $(r=0.7, p<0.05)$. The time required to achieve a complete clinical remission in patients with and without scalp involvement was $39.1 \pm 47.1$ and $9.1 \pm 7.8$ months, respectively. The duration of complete clinical remission was $14.1 \pm 17.4$ and $105.7 \pm 108.8$ months, respectively. The respective time required to achieve serological remission was $37.7 \pm 58.5$ and $15.5 \pm 18.8$ months, whereas the duration of serological remission was $9.2 \pm 18.8$ and $39.1 \pm 60.1$ months, respectively. The average concentration of anti-desmoglein 1 autoantibodies was significantly higher in patients with scalp involvement compared to patients without scalp involvement $(109.9 \pm 68.0 \mathrm{U} / \mathrm{ml}$ and $21.3 \pm 39.4 \mathrm{U} / \mathrm{ml})$.

Conclusions: Scalp involvement in pemphigus is associated with a higher disease severity, longer time required to achieve complete clinical and serological remission and may indicate the need for a more aggressive therapeutical approach.
\end{abstract}

Key words: desmoglein, hair follicle, hair, remission, therapy.

\section{Introduction}

Pemphigus is a group of autoimmune bullous diseases of the skin and mucous membranes characterized by the presence of autoantibodies targeting desmogleins (Dsg). According to literature data, scalp involvement is observed in $16-60 \%$ of patients, with a similar frequency in pemphigus vulgaris (PV) and pemphigus foliaceus (PF) [1-4]. The high frequency of scalp involvement in pemphigus may be associated with the expression of desmogleins in hair follicles [5]. It was reported that desmoglein 1 is located in the inner root sheath and the innermost layers of the outer root sheath, whereas the distribution of desmoglein 3 depends on the keratinization type and is expressed in all layers of the outer root sheath in the areas of trichilemmal keratinization and in the basal layers in the areas of epidermal keratinization $[6,7]$. Literature data show that scalp lesions may be long-lasting and resistant to treatment [8]. The possible significance of scalp involvement for the clinical course and immunopathological features of pemphigus has not been elucidated to date.

\section{Aim}

The aim of the study was to evaluate the clinical and prognostic significance of scalp involvement in the course of pemphigus.

\section{Material and methods}

A total of 75 patients with immunologically and histopathologically confirmed pemphigus (46 with PV and 29 with PF) were evaluated prospectively in this observational study. The patients were divided into two groups:

Address for correspondence: Malgorzata Olszewska MD, PhD, Department of Dermatology, Medical University of Warsaw, 82 A Koszykowa St, 02-008 Warsaw, Poland, phone: +48 2282422 00, +48 601214 715, e-mail: malgorzataolszewska@yahoo.com Received: 22.03.2017, accepted: 26.04.2017. 
patients with scalp involvement $(58 / 75,77.3 \%)$ and without scalp lesions (17/75, 22.7\%). The study groups did not differ significantly as regards age or sex. Initial treatment regimens were comparable in both groups, with the patients with PV receiving prednisone at the dose of $1.0 \mathrm{mg} / \mathrm{kg} /$ day with azathioprine at the dose of $1-2 \mathrm{mg} /$ $\mathrm{kg} /$ day, and the patients with PF being treated with prednisone at the dose of $0.5-0.75 \mathrm{mg} / \mathrm{kg} /$ day. The analysis included the evaluation of the sequence of appearance of pemphigus lesions. The clinical activity and damage at enrollment were evaluated using the Pemphigus Disease Area Index for the skin, scalp and mucous membranes $[9,10]$. Enzyme-linked immunosorbent assay was performed to assess anti-desmoglein 1 and anti-desmoglein 3 antibody concentration in the serum (Mesacup-2 Desmoglein Test, Nagoya, Japan, positive values $\geq 20 \mathrm{U} / \mathrm{ml}$ ). Indirect immunofluorescence with the use of monkey and guinea pig esophagus mucous membranes as substrates was performed to determine the titer of pemphigus antibodies.

The time needed to achieve complete clinical remission, the duration of complete clinical remission, the time needed to achieve serological remission as well as the duration of serological remission were analyzed. Complete clinical remission was defined, according to the consensus statement of 2008, as no new or established lesions in patients being off all systemic therapy for at least 2 months or patients receiving minimal therapy defined as less than or equal to $10 \mathrm{mg}$ /day of prednisone or prednisolone [11]. Serological remission was defined as the achievement of negative results in indirect immunofluorescence. Resistance to treatment of scalp lesions was defined as the maintenance of lesions in this loca- tion despite the resolution of lesions in other locations lasting for more than 2 months.

\section{Statistical analysis}

The results were analyzed using Statistica 12 software. Normality was tested using Shapiro-Wilk and Lilliefors tests. Mann-Whitney $U$ test was used to compare two study groups. The statistical significance level was set at $p<0.05$. Correlations between variables were tested using linear regression analysis. High-grade correlation was defined as $r$ (correlation coefficient) $>0.5$.

\section{Results}

\section{General data}

The group of patients with scalp involvement ( 28 women and 30 men) consisted of 30 patients with PV and 28 patients with PF. The average age was $59.4 \pm 16.2$ years. The average time from the diagnosis to enrollment was $68.5 \pm 93.5$ months.

The group without scalp involvement (12 women and 5 men) consisted of 16 patients with PV and 1 patient with PF. The average age was $56.2 \pm 17.1$ years. The average time from the diagnosis to enrollment was $72.4 \pm 82.4$ months.

\section{Scalp involvement in pemphigus}

Scalp involvement was observed in 30/46 (65.2\%) patients with PV and 28/29 (96.6\%) patients with PF.

The scalp was the first, second and third site of pemphigus in 8/58 (13.8\%), 23/58 (39.7\%) and 11/58 (19.0\%) cases, respectively. On average, scalp lesions occurred

Table 1. Disease characteristics in patients with pemphigus vulgaris and pemphigus foliaceus with scalp involvement

\begin{tabular}{|c|c|c|c|}
\hline Variable & $\begin{array}{l}\text { PV with scalp } \\
\text { involvement }\end{array}$ & $\begin{array}{l}\text { PF with scalp } \\
\text { involvement }\end{array}$ & $\begin{array}{c}\text { Statistical significance of } \\
\text { differences between PV and PF } \\
\text { with scalp involvement }(p<0.05)^{\star}\end{array}$ \\
\hline $\begin{array}{l}\text { Development of scalp lesions } \\
\text { [months after disease onset] }\end{array}$ & $\begin{array}{c}25.3 \pm 41.2 \\
(n=28)\end{array}$ & $\begin{array}{l}7.0 \pm 12.5 \\
(n=27)\end{array}$ & 0.01 \\
\hline PDAI scalp activity score- 0 & $8 / 30(26.7 \%)$ & $2 / 28(7.1 \%)$ & 0.1 \\
\hline PDAI scalp activity score-1 & $5 / 30(16.7 \%)$ & $3 / 28(10.7 \%)$ & 0.57 \\
\hline PDAI scalp activity score-2 & $3 / 30(10.0 \%)$ & $5 / 28(17.9 \%)$ & 0.45 \\
\hline PDAI scalp activity score-3 & 4/30 (13.3\%) & $2 / 28(7.1 \%)$ & 0.49 \\
\hline PDAI scalp activity score-4 & $2 / 30(6.7 \%)$ & $9 / 28(32.1 \%)$ & 0.04 \\
\hline PDAI scalp activity score-10 & $8 / 30(26.7 \%)$ & $7 / 28(25.0 \%)$ & 0.91 \\
\hline Scalp as the first location & $3 / 30(10.0 \%)$ & $5 / 28(17.9 \%)$ & 0.45 \\
\hline Scalp as the second location & $11 / 30(36.7 \%)$ & $12 / 28(42.9 \%)$ & 0.75 \\
\hline Scalp as the third location & $7 / 30(23.3 \%)$ & $4 / 28(14.3 \%)$ & 0.47 \\
\hline Scalp lesions resistant to treatment & $20 / 30(66.7 \%)$ & $19 / 28(67.9 \%)$ & 0.97 \\
\hline
\end{tabular}

PV-pemphigus vulgaris, PF - pemphigus foliaceus, PDAI - Pemphigus Disease Area Index at enrollment, "statistical significance at $p<0.05$. 
$16.3 \pm 31.9$ months after the onset of disease, significantly later in PV $(25.3 \pm 41.2$ months $)$ in comparison to PF (7.0 \pm 12.5 months; $p=0.01)$. In $34 / 58$ (58.6\%) patients, the scalp was the site of persistent residual lesions. Scalp lesions were resistant to treatment in 39/58 (67.2\%) patients.

In the group of patients with scalp involvement, the average Pemphigus Disease Area Index (PDAI) scalp activity score was $3.7 \pm 4.1$ in PV and $4.8 \pm 3.4$ in PF $(p=0.12)$.

The details are shown in Table 1.

\section{Disease severity in patients with scalp involvement}

The group of patients with scalp involvement significantly differed from the group of patients without scalp involvement in terms of PDAI skin activity score, PDAl skin damage score and PDAI mucosa activity score (Table 2). The respective variables were $8.3 \pm 7.7$ and 2.8 \pm 6.1 for PDAl skin activity score, $1.2 \pm 1.8$ and $0.2 \pm 1.0$ for PDAl skin damage score, $2.7 \pm 8.2$ and $9.1 \pm 15.5$ for PDAl mucosa activity score.

A positive correlation between PDAI scalp activity score and PDAl skin activity score was observed $(r=0.7$, $p<0.05$ ) (Figure 1).

Statistically significant differences between groups of pemphigus patients with and without scalp involvement were also observed in terms of such variables as the average time needed to achieve complete clinical remission (39.1 \pm 47.0 months vs. $9.1 \pm 7.8$ months; $p=$ $0.02)$, the average duration of complete clinical remission (14.1 \pm 17.4 months vs. $105.7 \pm 108.8$ months, $p=0.03$ ), the average time needed to achieve serological remission (37.7 \pm 58.5 months vs. $15.5 \pm 18.8$ months; $p=0.01$ ) and the average duration of serological remission $(9.2 \pm 18.8$ months vs. $39.1 \pm 60.1$ months; $p=0.03$ ).

In the group of patients with scalp involvement, the time needed to achieve complete clinical remission was $44.7 \pm 53.3$ months in PV and $29.0 \pm 32.5$ months in PF. The average duration of complete clinical remission was 15.2 \pm 21.8 months in PV and $12.6 \pm 8.9$ months in PF. The average time needed to achieve serological remission was $47.8 \pm 71.3$ months and $20.4 \pm 19.8$ months, respectively. The average duration of serological remission was 12.5 \pm 24.3 months and $5.6 \pm 8.7$ months, respectively. The differences were not statistically significant. Patients with $P V$ and PF with scalp involvement differed significantly in terms of PDAI skin activity score ( $5.8 \pm 7.6$ vs. $11.0 \pm 7.6$; $p=0.005)$.

Detailed data are shown in Table 2.

\section{Pemphigus antibodies}

The group of patients with scalp involvement and without scalp involvement significantly differed in terms of the highest median titer on guinea pig esophagus, which was 160 (0-5120) and 40 (0-320), respectively.

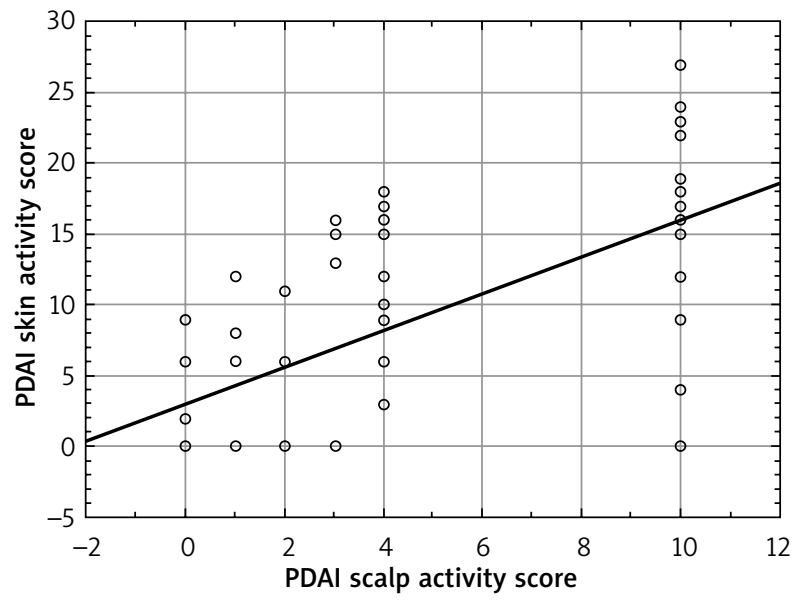

Figure 1. Correlation between PDAI scalp activity score and PDAl skin activity score at enrollment

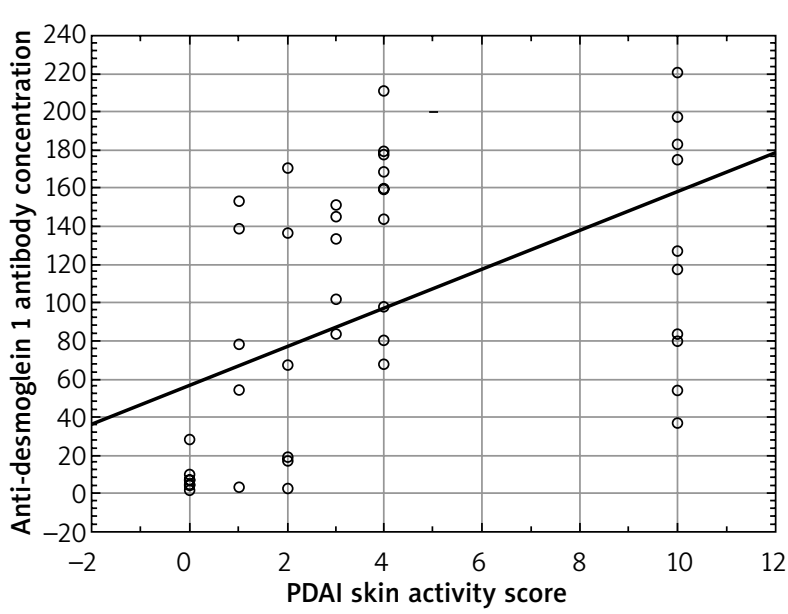

Figure 2. Correlation between PDAI scalp activity score and anti-desmoglein 1 antibody concentration at enrollment

The average concentration of anti-Dsg1 autoantibodies was $109.9 \pm 68.0 \mathrm{U} / \mathrm{ml}$ in the group of patients with scalp involvement and $21.3 \pm 39.4 \mathrm{U} / \mathrm{ml}$ in the group without scalp involvement $(p=0.003)$.

The average concentration of anti-desmoglein 1 autoantibodies correlated positively with PDAI scalp activity score $(r=0.7, p<0.05)$ (Figure 2 ).

Table 2 summarizes the differences between groups of patients with and without scalp involvement in the course of pemphigus.

\section{Discussion}

The frequency of scalp involvement in the course of pemphigus is estimated at 16-60\% [1-4]. The scalp is the second (after oral mucosa) or the third most common location (after mucous membranes and trunk) of PV $[5,9,12]$. Arya et al. reported the prevalence of scalp involvement separately for both types of pemphigus be- 


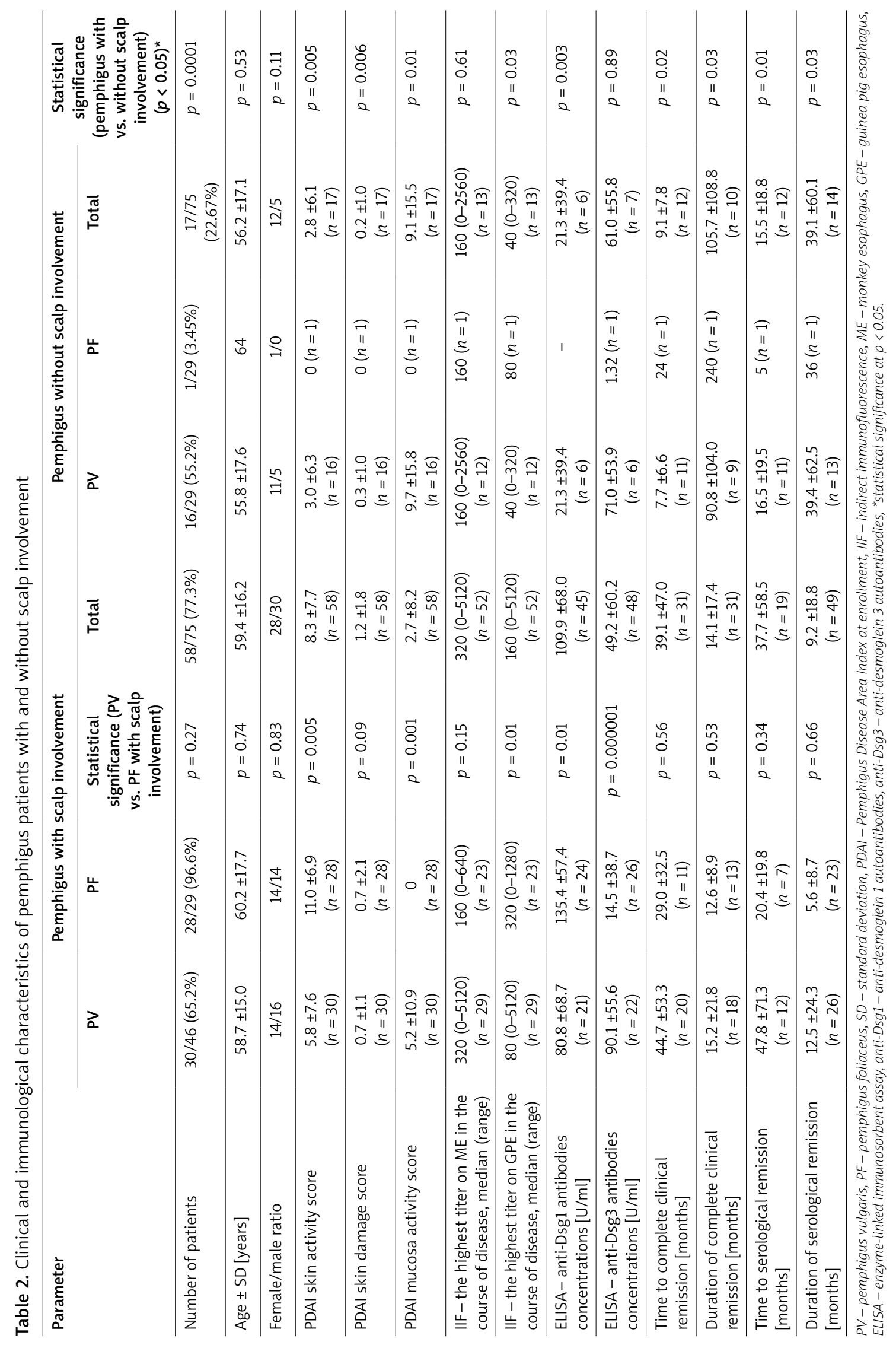


ing $18.6 \%$ in PV and $16 \%$ in PF [4]. Our results show that the frequency of scalp involvement in patients with pemphigus is $77.3 \%$. The frequency was higher in PF (96.6\%) than in PV (65.2\%). According to literature data, the scalp is the first location in $9-15 \%$ of patients with pemphigus $[2,3,13]$. In our group, the scalp was the first location of pemphigus in $8 / 75(10.7 \%)$ cases, which is consistent with the previous data. Furthermore, in 23/75 (30.7\%) patients, the scalp was the second location and in 11/75 (14.6\%), it was the third site of pemphigus lesions. Scalp lesions occurred earlier in the course of PF in comparison with $\mathrm{PV}$, which has not been reported before.

The high prevalence of scalp involvement in pemphigus may be associated with antigen load in this area [5]. Several studies showed that the scalp, and hair follicles in particular, are the site of a significant concentration of PV [12] and PF antigens [14]. The presence of desmogleins in hair follicles was investigated in detail [12]. These data show that desmoglein 1 is expressed in the inner root sheath and the innermost layers of the outer root sheath as well as epidermal suprabasal cells. Desmoglein 2, an antigen of a less prominent role in pemphigus, is highly expressed in the least differentiated cells of the cutaneous epithelium, including the hair follicle bulge of the fetus and adult, bulb matrix cells and the basal layer of the outer root sheath. The expression of desmoglein 3 in the hair follicle depends on the keratinization type. In the areas of trichilemmal keratinization of the hair follicle, desmoglein 3 is expressed throughout all layers of the outer root sheath, while in the areas of epidermal keratinization, its expression is limited mainly to the basal layer. Moreover, a strong expression of Dsg3 is detected in the medulla. Desmoglein 4 is mainly expressed in the hair shaft cortex, the lower hair cuticle and the upper inner root sheath $[6,15]$. Some authors suggested that desmoglein 4 may play a role as an additional autoantigen in PV [6].

Scalp is rarely the site of isolated pemphigus lesions. Cases of unilesional PV of the scalp have been described previously, with one of them developing at the site of a surgical trauma $[16,17]$. Isolated scalp erosions have also been reported in PF [18]. Furthermore, cases of pemphigus vegetans confined to the scalp were described in the literature [19-21]. In our group we did not observe patients with scalp involvement as the sole manifestation throughout the course of pemphigus. However, we have observed that in $58.6 \%$ of the patients with scalp involvement, the scalp was the site of residual pemphigus lesions.

Scalp lesions in pemphigus tend to be recalcitrant [8]. In our study the resistance to treatment, defined as the maintenance of scalp lesions despite resolving lesions in other locations, was $67.2 \%$ and was comparable in both types of pemphigus.

To our knowledge, to date there have been no reports on scalp involvement in pemphigus basing on an objective evaluation of the severity of scalp lesions in pem- phigus. In our study we used PDAI to assess the activity and damage of skin and scalp involvement as well as the activity of mucosa involvement [9]. Our results show that the severity of scalp involvement is positively correlated with the severity of skin involvement.

Indirect immunofluorescence and enzyme-linked immunosorbent assay are useful tools in monitoring pemphigus activity [22, 23]. In the study of Maragno et al. performed in 18 patients with PV and 20 patients with PF, direct immunofluorescence revealed predominance of IgG4 and IgG1 deposits in the hair follicles, independently from disease activity [24]. To our knowledge, thus far there have been no data addressing the correlation of the serum autoantibody profile and activity of scalp lesions in patients with pemphigus. Our study results show that patients with scalp involvement have higher median autoantibody titers on guinea pig esophagus as well as higher serum concentration of anti-desmoglein 1 autoantibodies. Furthermore, a positive correlation between PDAI scalp activity and anti-Dsg1 autoantibodies levels was observed.

There are currently no literature data addressing the possible association of scalp involvement with clinical and serological remission. Our results show that scalp involvement in the course of pemphigus is associated with a higher general disease severity, a longer time required to achieve complete clinical remission, and a shorter duration of complete clinical remission. The achievement of serological remission, defined as the negative result of indirect immunofluorescence on monkey and guinea pig esophagus, was delayed and the duration of serological remission was significantly shortened.

\section{Conclusions}

Our results show that scalp involvement may be a marker of a severe course of pemphigus. Therefore, it may be hypothesized that patients with scalp involvement should receive a more intensive immunosuppressive therapy to achieve clinical and serological remission.

\section{Acknowledgments}

We thank Professor Leszek Pączek for his kind support and making his labs accessible for this study. This study was supported by research grant from the Warsaw Medical University no. 1M4/PM11D/14.

\section{Conflict of interest}

The authors declare no conflict of interest.

\section{References}

1. Coronel-Perez IM, Rodriguez-Rey EM, Perez-Bernal AM et al. [Epidemiology of pemphigus in the Hospital Universitario 
Virgen Macarena, Seville, Spain, 2005-2006]. Actas Dermosifiliogr 2009; 100: 121-5.

2. Chams-Davatchi C, Valikhani M, Daneshpazhooh M, et al. Pemphigus: analysis of 1209 cases. Int J Dermatol 2005; 44: 470-6.

3. Esmaili N, Chams-Davatchi C, Valikhani M, et al. Pemphigus vulgaris in Iran: a clinical study of 140 cases. Int I Dermatol 2007; 46: 1166-70.

4. Arya SR, Valand AG, Krishna K. A clinico-pathological study of 70 cases of pemphigus. Indian J Dermatol Venereol Leprol 1999; 65: 168-71.

5. Wilson CL, Dean D, Wojnarowska F. Pemphigus and the terminal hair follicle. J Cutan Pathol 1991; 18: 428-31.

6. Kljuic A, Bazzi H, Sundberg JP, et al. Desmoglein 4 in hair follicle differentiation and epidermal adhesion: evidence from inherited hypotrichosis and acquired pemphigus vulgaris. Cell 2003; 113: 249-60.

7. Wu H, Stanley JR, Cotsarelis G. Desmoglein isotype expression in the hair follicle and its cysts correlates with type of keratinization and degree of differentiation. J Invest Dermatol 2003; 120: 1052-7.

8. Rackett SC, Rothe MJ, Hoss DM, et al. Treatment-resistant pemphigus vegetans of the scalp. Int I Dermatol 1995; 34: 865-6.

9. Rahbar Z, Daneshpazhooh M, Mirshams-Shahshahani M, et al. Pemphigus disease activity measurements: pemphigus disease area index, autoimmune bullous skin disorder intensity score, and pemphigus vulgaris activity score. JAMA Dermatol 2014; 150: 266-72.

10. Daniel BS, Hertl M, Werth VP, et al. Severity score indexes for blistering diseases. Clin Dermatol 2012; 30: 108-13.

11. Murrell DF, Dick S, Ahmed AR, et al. Consensus statement on definitions of disease, end points, and therapeutic response for pemphigus. J Am Acad Dermatol 2008; 58: 1043-6.

12. Sison-Fonacier L, Bystryn JC. Regional variations in antigenic properties of skin. A possible cause for disease-specific distribution of skin lesions. J Exp Med 1986; 164: 2125-30.

13. Salmanpour R, Shahkar H, Namazi MR, et al. Epidemiology of pemphigus in south-western Iran: a 10-year retrospective study (1991-2000). Int J Dermatol 2006; 45: 103-5.

14. Ioannides D, Hytiroglou P, Phelps RG, et al. Regional variation in the expression of pemphigus foliaceus, pemphigus erythematosus, and pemphigus vulgaris antigens in human skin. J Invest Dermatol 1991; 96: 159-61.

15. Bazzi H, Getz A, Mahoney MG, et al. Desmoglein 4 is expressed in highly differentiated keratinocytes and trichocytes in human epidermis and hair follicle. Differentiation 2006; 74: 129-40.

16. Ferrara G, Massone C, Zalaudek I, et al. Unilesional pemphigus vulgaris of the scalp. Dermatol Online J 2009; 15: 9.

17. Oretti G, Giordano D, Di Lella F, et al. Unilesional pemphigus vulgaris of the scalp after cochlear implantation. Am J Otolaryngol 2011; 32: 80-1.

18. Tyros G, Kalapothakou K, Christofidou E, et al. Successful treatment of localized pemphigus foliaceus with topical pimecrolimus. Case Rep Dermatol Med 2013; 2013: 489618.

19. Mori M, Mariotti G, Grandi V, et al. Pemphigus vegetans of the scalp. J Eur Acad Dermatol Venereol 2016; 30: 368-70.

20. Danopoulou I, Stavropoulos P, Stratigos A, et al. Pemphigus vegetans confined to the scalp. Int J Dermatol 2006; 45: 1008-9.

21. Lehrhoff S, Miller K, Fischer M, et al. Localized pemphigus with vegetative features. Dermatol Online J 2012; 18: 11
22. Daneshpazhooh M, Chams-Davatchi C, Khamesipour A, et al. Desmoglein 1 and 3 enzyme-linked immunosorbent assay in Iranian patients with pemphigus vulgaris: correlation with phenotype, severity, and disease activity. J Eur Acad Dermatol Venereol 2007; 21: 1319-24.

23. Weiss D, Ristl R, Griss J, et al. Autoantibody levels and clinical disease severity in patients with pemphigus: comparison of aggregated anti-desmoglein ELISA values and indirect immunofluorescence titres. Acta Derm Venereol 2015; 95 : 559-64.

24. Maragno L, Bussato WM, Maruta CW, et al. Characterization of the humoral and in situ autoantibody profile of scalp involvement in pemphigus. J Eur Acad Dermatol Venereol 2016; 30: e57-9. 